\title{
TEM Study of Manganese Biosorption by Cyanobaterium Synechocystis 6803
}

\author{
Alice Dohnalkova*, Christina Bilskis** and David W. Kennedy** \\ *W. R. Wiley Environmental Molecular Sciences Laboratory, ** Environmental Microbiology, \\ Pacific Northwest National Laboratory, P.O. Box 999, K8-93, Richland, WA 99354
}

The capture of solar energy and its conversion into chemical energy in photosynthetic organisms involves a series of charge reactions across photosynthetic membranes. Oxygen is generated by a proton-electron coupling in photosystem II (PSII) during a water oxidation process where hydrogen is extracted from water terminally bound to a $\mathrm{Mn}_{4} \mathrm{Ca}_{1} \mathrm{Cl}_{\mathrm{x}}$ inorganic cluster [1]. Manganese is, therefore, an essential catalytic element for photosynthetic growth in cyanobacteria and plants. Since bioavailability of this micronutrient largely depends on the Mn concentration in natural environments, cells have to manage its uptake in order to endure Mn fluctuations. Previous studies have shown that metal biosorption in cyanobacteria can occur by passive adsorption to their outer membrane (pool A), and by metabolically mediated internal uptake [2]. The fresh water cyanobacterium Synechocystis 6803 has been widely used as a model organism for studying photosynthetic processes. This Gram-negative organism has an intricate architecture of internal thylakoid membranes where photosynthetic electron transfer takes place. Here we report on the spatial distribution of Mn biosorbed by cells in both external pool A and intracellular pool B, as observed and analyzed by methods of TEM.

The Synechocystis 6803 cells were cultured in BG11 medium at $30^{\circ} \mathrm{C}$ with continuous irradiance and constant air bubbling. To determine the influence of solid or liquid Mn substrate and its oxidation state on the cell biosorption ability, cells were exposed to two Mn substrates: $1 \mathrm{mM}$ solution of $\mathrm{MnCl}_{2}$, and $0.5 \mathrm{mM}$ suspension of nanocrystalline $\mathrm{MnO}_{2}$. Cells were incubated with the respective Mn solutions for 48 hours, harvested, and processed using a modified protocol for plastic embedding of bacterial samples containing minerals that was developed in our laboratory [3]. In order to preserve the fragile redox conditions within the cells, all the common heavy metal-based fixatives and stains were omitted, resulting in cells with very low contrast produced principally by electron-dense manganese precipitates. Thin sections were imaged and analyzed using JEOL 2010 HRTEM coupled with EDS (Oxford) and EELS (Gatan) systems.

Manganese uptake was measured using a colorimetric method. Cells incubated with Mn solutions were able to take up about 150uM of Mn(II) or Mn(IV) in 48 hours. The predominant accumulation of Mn was associated with the outer membrane for both Mn substrates. Massive deposits seemed to be related in a large extent to the external polymeric substances (EPS) as shown in Fig. 1A-C. Elemental analyses of these precipitates revealed a signal consistent with manganese phosphate. The potential of EPS such as polysaccharides for biosorption or reduction of metals has been described [4], however, the fact that Mn bound to the EPS withstood multiple washes during TEM sample processing is remarkable. From our work with Gram-negative soil bacteria, we hypothetized that the periplasm, an area between the outer and plasma membrane, might be the storage space for internal $\mathrm{Mn}$ in pool B. This phenomenon was 
not observed at any time point for either culture exposed to the Mn. Instead, thin layers of $\mathrm{Mn}$ deposits were often found lining the outer and plasma membrane (F). In the $\mathrm{MnCl}_{2}$ solution only, we also observed fine deposits of Mn precipitates along the thylakoid membranes deep inside the cells (Fig. E).

Localization of Mn precipitation sites in Synechocystis has important implications for better understanding of the Mn transport and storage processes within cyanobacterial cells, as well as of metal precipitation, solubilization and cycling in the environment.

\section{References}

[1] W. Hillier and G.T. Babcock, Plant Physiol. 125 (2001) 33-37. [2] Keren N. et al., Biochemistry 41: 15085-15092 (2002). [3] Dohnalkova et al. Microsc. Microanal.7, Suppl 2, $756-757$ (2001). [4] Parker D.L. et al., J. Appl. Phycology 12: 219-224 (2000). This work was conducted at the W. R. Wiley Environmental Molecular Sciences Laboratory (EMSL), under the LDRD Program at the Pacific Northwest National Laboratory, a multiprogram national laboratory operated by Battelle for the U.S. DOE under Contract DE-AC05-76RLO1830.

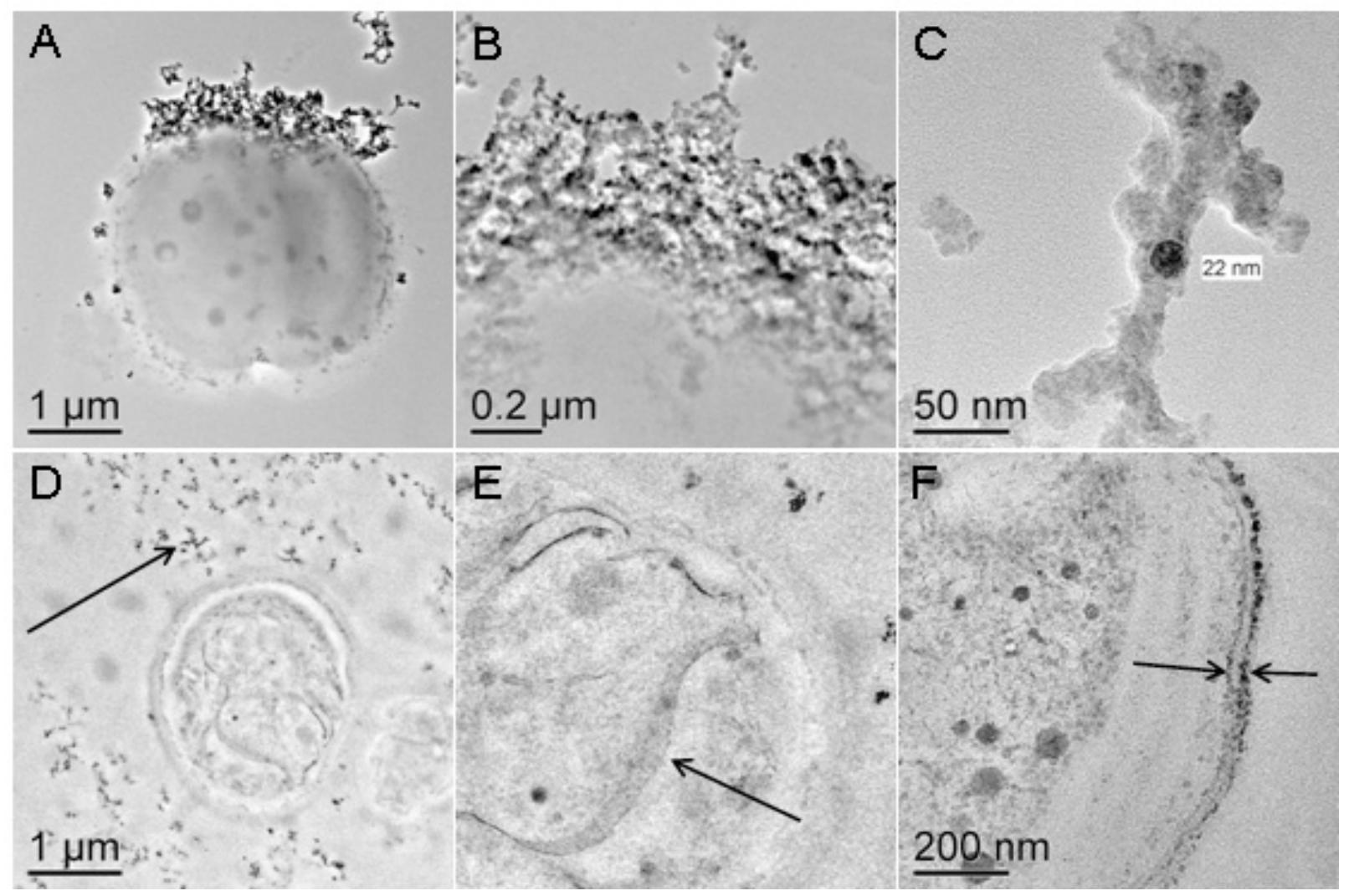

Fig.1. TEM images of a whole mount of Synechocystis exposed to $1 \mathrm{mM} \mathrm{MnCl} 2$ for 48 hours.

Precipitated material is associated with the outer membrane, possibly bound to the EPS (A, B). The material appears to be poorly nanocrystalline, containing predominantly $\mathrm{Mn}$ and $\mathrm{P}$ (C). TEM images of thin sections of cells from the same experiment, revealing the internal deposition of $\mathrm{Mn}$ (pool B), as well as a dispersed material outside of the cells (pool A - arrow in D.) Very thin deposits of Mn along thylakoid membranes are apparent (E). Mn precipitates on plasma and outer membranes were found in $\mathrm{MnO}_{2}$ treatment (F). 